\title{
Phase Misalignment between Suprachiasmatic Neuronal Oscillators Impairs Photic Behavioral Phase Shifts But Not Photic Induction of Gene Expression
}

\author{
Michael D. Schwartz, Seth Congdon, and Horacio 0. de la Iglesia \\ Department of Biology and Program of Neurobiology and Behavior, University of Washington, Seattle, Washington 98195
}

The ability of the circadian pacemaker within the suprachiasmatic nucleus (SCN) to respond to light stimulation in a phase-specific manner constitutes the basis for photic entrainment of circadian rhythms. The neural basis for this phase specificity is unclear. We asked whether a lack of synchrony between SCN neurons, as reflected in phase misalignment between dorsomedial ( $\mathrm{dmSCN}$ ) and ventrolateral (vISCN) neuronal oscillators in the rat, would impact the ability of the pacemaker to respond to phase-resetting light pulses. Light pulses delivered at maximal phase misalignment between the vlSCN and dmSCN oscillators increased expression of Perl mRNA, regardless of the circadian phase of the dmSCN. However, phase shifts of locomotor activity were only observed when the vlSCN and dmSCN were phase aligned at the time of stimulation. Our results fit a model in which a vISCN oscillator phase gates its own response to light and in turn relays light information to a $\mathrm{dmSCN}$ oscillator. This model predicts that the phase misalignment that results from circadian internal desynchronization could preserve the ability of light to induce gene expression within the master circadian clock but impair its ability to induce behavioral phase shifts.

\section{Introduction}

In mammals, circadian rhythms are coordinated by a pacemaker within the hypothalamic suprachiasmatic nucleus (SCN) (Klein et al., 1991). Within the SCN, rhythmicity arises from multiple cell-autonomous circadian oscillators (Welsh et al., 1995) that through their coupled activity constitute a tissue pacemaker with a coherent periodic output (Reppert and Weaver, 2002; Aton and Herzog, 2005). Although potentially all SCN neurons are singlecell oscillators (Welsh et al., 1995; Webb et al., 2009), distinct subpopulations can be differentiated via neurochemistry, connectivity, and gene expression (Morin, 2007). In rats, light cues reach the SCN via direct inputs to the ventrolateral (vl) SCN (Moore and Lenn, 1972; Moore et al., 2002; Morin and Allen, 2006). The vlSCN in turn innervates the dorsomedial (dm) SCN with sparse reciprocal innervation (Leak et al., 1999). This segregation of SCN afferents, intrinsic connections, and efferents (Leak and Moore, 2001) suggests that the dmSCN and vlSCN play distinct roles in the entrainment of circadian rhythmicity and the regulation of circadian outputs.

Recently we reported that, when housed in symmetrical $11 \mathrm{~h}$ light/dark cycles (LD22), rats exhibit simultaneous entrained and dissociated locomotor activity rhythms (Campuzano et al., 1998; de la Iglesia et al., 2004b) that are correlated with independent

Received April 12, 2010; revised Aug. 2, 2010; accepted Aug. 3, 2010.

This research was supported by National Institutes of Health Grant R01MH075016 (H.O.d.I.I.) and the Amgen Scholars Program (S.C.). We thank Dr. Cheryl Wotus, Yoshito Kosai, and Benjamin Smarr for technical assistance.

Correspondence should be addressed to Horacio 0. de la Iglesia, University of Washington, Department of Biology, 24 Kincaid Hall, Box 351800, Seattle, WA 98195. E-mail: horaciod@u.washington.edu.

M. D. Schwartz's present address: Department of Pharmacology and Experimental Therapeutics, University of Maryland School of Medicine, Baltimore, MD 21201

DOI:10.1523/JNEUROSCI.1853-10.2010

Copyright $\odot 2010$ the authors $\quad 0270-6474 / 10 / 3013150-07 \$ 15.00 / 0$ clock gene oscillations in the vlSCN and dmSCN, respectively (de la Iglesia et al., 2004b; Lee et al., 2009). In these forced desynchronized rats, pineal melatonin, body temperature, and rapid-eye movement (REM) sleep rhythms also dissociate from slow-wave sleep rhythms (Cambras et al., 2007; Lee et al., 2009; Schwartz et al., 2009), suggesting that the two SCN subregions can act as independent pacemakers driving distinct physiological rhythms (Schwartz, 2009). The stable dissociation between vlSCN and dmSCN oscillators offers a unique opportunity to assess the role of SCN organization in decoding photic information. Light pulses presented during the subjective night, but not during the subjective day, rapidly induce expression of the immediate early gene c-fos (Aronin et al., 1990; Kornhauser et al., 1990; Rusak et al., 1990; Schwartz et al., 2000) and the clock gene Per1 (Shigeyoshi et al., 1997; Miyake et al., 2000) within the retinorecipient vlSCN (Yan et al., 1999; Schwartz et al., 2000), resulting in phase shifts of behavioral circadian rhythms (Daan and Aschoff, 2001; Johnson et al., 2003). This circadian-gated photic induction of Perl is thought to be a critical component of the phaseresetting mechanism, but it is not clear how the SCN pacemaker restricts the induction of gene expression and the phase resetting of circadian behavioral rhythms to the subjective night. To address this question, we tested whether classical responses to phase-resetting light pulses were preserved in forced desynchronized rats. We found that, although photic induction of Perl in the vlSCN was not modulated by the circadian phase of the dmSCN, changes in the phase of the locomotor activity rhythm were only observed when both vlSCN and dmSCN phases were aligned. These results support a model in which the vlSCN and dmSCN are individual oscillators, and photic cues are first intrinsically decoded by the vlSCN and then relayed to the dmSCN. 

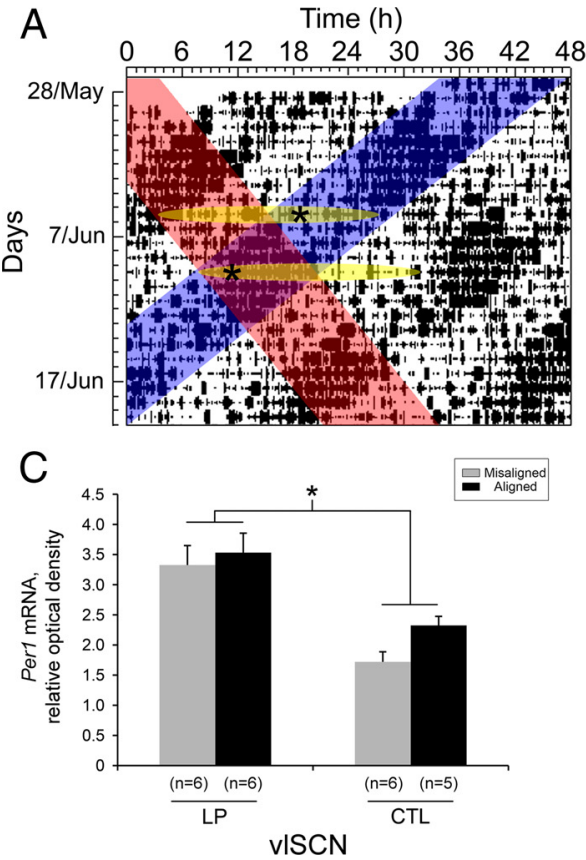

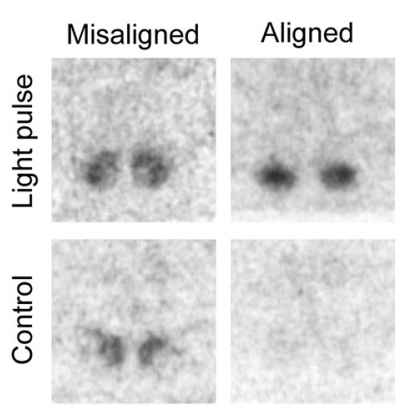

D

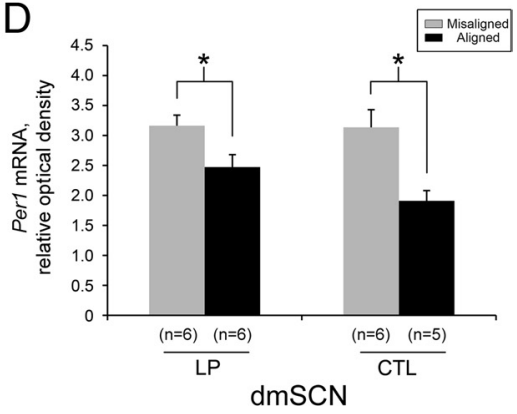

Figure 1. Light pulses induce Per1 expression within the vISCN regardless of the dmSCN circadian phase. $A$, Representative double-plotted actogram from a rat maintained under LD22, showing the entrained locomotor bout (blue highlight) and the dissociated locomotor bout (red highlight). Yellow ovals indicate aligned phase (bottom oval) and misaligned phase (top oval). Asterisks indicate time of light pulse or control dark pulse. $\boldsymbol{B}-\boldsymbol{D}$, Representative autoradiographs $(\boldsymbol{B})$ and relative optical densities $(\boldsymbol{C}, \boldsymbol{D})$ of Per1 mRNA expression in the SCN of forced desynchronized rats after a 30 min light pulse (LP) or control dark pulse (CTL) on the day of maximal phase misalignment or alignment. C, Per1 mRNA expression in the vISCN; ${ }^{*} p<0.0001$. D, Per1 mRNA expression in the dmSCN; ${ }^{*} p=0.0004$.

\section{Materials and Methods}

Animals. Male Wistar rats (Charles River) were singly housed in polycarbonate cages $(20 \times 25 \times 22 \mathrm{~cm})$ in light- and sound-protected isolation chambers with ad libitum access to food and water and constant temperature. Unless otherwise noted, animals were maintained under a symmetrical $11 \mathrm{~h}$ LD cycle; illumination was provided by white fluorescent tubes (100-150 lux at cage level) mounted above each row of cages, with dim red light ( $<2$ lux) during the dark phase of the LD cycle and during constant darkness (DD). Locomotor activity was continuously monitored via paired crossed infrared photobeam detectors connected to a personal computer running the Clocklab data acquisition system (Actimetrics). All experiments were conducted at the University of Washington and were performed in compliance with the University of Washington Animal Care and Use Committee and National Institutes of Health Guide for the Care and Use of Laboratory Animals.

Experimental design. Animals were monitored in LD22 for 2-3 weeks until locomotor activity rhythms desynchronized. Desynchrony was first verified by visual inspection of the actograms. Then, using $\chi^{2}$ periodogram analysis, which detects statistically significant oscillations of specific periods (Sokolove and Bushell, 1978), we confirmed that two significant periods ( $\alpha=0.05$ ) were observed [one at $22.0 \mathrm{~h}$ (Fig. $1 \mathrm{~A}$, blue) and the other being $>24$ h (Fig. $1 A$, red)]. After desynchronization was confirmed statistically for each animal, two independent investigators traced the onset of locomotor activity for the dissociated bout, based on the same 2-3 weeks of data used for the periodogram analysis. All light treatments occurred $3.8 \mathrm{~h}$ after lights off, on a day in which the dissociated activity bout fully coincided with the dark phase ["aligned" phase (Fig. $1 A$, bottom oval, pulse indicated by asterisk)] or on a day in which the dissociated bout fully occurred in the light phase ["misaligned" phase (Fig. $1 A$, top oval)]. In 22LD, $3.8 \mathrm{~h}$ represents $\sim 4$ Zeitgeber hours, namely one- sixth of the full cycle. We reasoned that a light pulse at this time would be equivalent to applying a pulse $4 \mathrm{~h}$ after lights off under a $24 \mathrm{~h} \mathrm{LD}$ cycle, which leads to predictable phase delays in the rat (Summer et al., 1984). Animals were moved in the dark from their home chamber to a second chamber, where they were either exposed to a $30 \mathrm{~min}$ bright light pulse (1000 lux) or control dark pulse (dim red light only). Animals were left in their cages for the duration of the pulse. At the conclusion of the pulse, animals were either returned to their home chamber or immediately released into DD.

In situ hybridization. At 30 min after return to home chamber (60 min after the start of the light pulse), animals were decapitated under dim red light, and brains were rapidly removed and frozen in 2-methylbutane cooled to $-30^{\circ} \mathrm{C}$. Coronal sections, $16 \mu \mathrm{m}$ thick, through the SCN were cut on a cryostat and mounted onto slides coated with Vectabond (Vector Laboratories).

Linearized recombinant plasmids were used to generate an antisense cRNA probe for rat Per1 (plasmid generously provided by Dr. H. Okamura, Kyoto University, Kyoto, Japan). Probes were transcribed in the presence of $\left[{ }^{35}\right.$ S $]$ UTP with T7 RNA polymerase, using a MaxiScript in vitro transcription kit (Ambion), and in situ hybridization was performed as described previously (de la Iglesia, 2007). Briefly, slides were air dried, fixed in $4 \%$ paraformaldehyde, treated with acetic anhydride, delipidated in an ethanol and chloroform sequence, and allowed to air dry. Once fully dry, slides were prehybridized for $1 \mathrm{~h}$ in $50 \% 4 \times$ SSC/ $50 \%$ deionized formamide at $37^{\circ} \mathrm{C}$ and then hybridized in humidified chambers overnight with the cRNA probe in hybridization buffer containing dextran sulfate, deionized formamide, SSC, Denhardt's solution, tRNA, and sheared single-stranded DNA. Control slides in which probe was omitted from the hybridization did not yield signal (data not shown). After hybridization, slides were rinsed in $1 \times$ SSC and incubated multiple times in $50 \%$ formamide at $52^{\circ} \mathrm{C}$, RNase A (Sigma) for $30 \mathrm{~min}$ at $37^{\circ} \mathrm{C}$ and again in $50 \%$ formamide at $52^{\circ} \mathrm{C}$. Slides were then washed in sequential ethanol series and air dried. Autoradiographic films were generated by exposing slides to Ultramax film (Kodak) in light-tight cartridges for $72 \mathrm{~h}$.

Autoradiographic films were scanned and stored as high-resolution grayscale TIFF files. Optical densities (ODs) were measured in NIH Image J. Templates were made for the right and left $\mathrm{dmSCN}$ and vlSCN by taking the best-fit outline from several representative sections picked at random from the experimental sample (Fig. $1 B$ ). Background grayscale values were taken within a circle placed in the adjacent hypothalamus of each section. Relative OD was calculated as the grayscale value for the region of interest divided by the background grayscale value for the same section; this normalized OD was measured bilaterally for each subregion in four sections and averaged for each animal.

Immunohistochemistry. To assess c-Fos immunoreactivity (IR) in the same tissue that was processed for in situ hybridization, we modified a fresh-frozen immunohistochemistry protocol (Sundquist and Nisenbaum, 2005). Unless otherwise indicated, incubations were conducted for $1 \mathrm{~h}$ at room temperature with light agitation, antisera and blocking agents were made in Tris-buffered saline with $0.05 \%$ Triton $\mathrm{X}-100, \mathrm{pH}$ 7.2 (TBS-T), with $1 \%$ bovine serum albumin (Sigma), and tissue was rinsed in TBS-T. Fresh-frozen brains were sectioned through the SCN as described above. Sections were thawed at room temperature and then fixed in $4 \%$ paraformaldehyde for $10 \mathrm{~min}$, rinsed in TBS-T, and then incubated in methanol containing $0.3 \%$ hydrogen peroxide for $15 \mathrm{~min}$. Sections were rinsed in TBS-T and incubated in the following: (1) $10 \%$ normal goat serum (Vector Laboratories); (2) rabbit anti-c-Fos antiserum (1:2000; Santa Cruz Biotechnology) overnight at $4^{\circ} \mathrm{C}$; (3) goat anti-rabbit antiserum (1:200; Vector Laboratories); and (4) avidin-biotin complex (Vector Laboratories). Sections were then rinsed and reacted in VIP chromagen (Vector Laboratories) to visualize immunoreactive cells. Sec- 
tions were rinsed in $\mathrm{dH}_{2} \mathrm{O}$, ethanol, and xylene and coverslipped with Permount (Vector Laboratories).

c-Fos-immunoreactive cell counts were made by an observer blind to treatment groups. Photomicrographs of the whole SCN in three sections were taken at $20 \times$. Templates for the right and left dmSCN and vlSCN were made from these images as described above. Because of the small number of cells visible in the thin sections, all c-Fos-immunoreactive cells within the boundaries of each template were counted and totaled for each animal. Control experiments in which the primary antibody was omitted from the antiserum resulted in loss of stain (data not shown).

Assessment of phase shifts. After light treatment, animals were monitored in DD (constant dim red light, $<2$ lux) for at least 2 weeks. All measurements of phase were made by eye-fitting activity onset for a 1-2 week period in DD beginning $7 \mathrm{~d}$ after light treatment and extrapolating this eye-fitted onset back to the day of treatment. Eye-fit onsets were plotted independently by two investigators blind to the treatment groups and were in agreement with each other (data not shown). After release into constant conditions from LD22, locomotor rhythms rapidly fuse into a single bout (Campuzano et al., 1998; de la Iglesia et al., 2004b). To determine whether the initial phase of this fused activity bout was tied to one of the two desynchronized bouts, the onset of the fused bout in all control-pulsed animals was referenced to that of (1) the dissociated bout and (2) the entrained bout (which coincided with lights off). The extrapolated fused onsets were then plotted relative to the 22LD cycle and relative to the $\sim 25 \mathrm{~h}$ dissociated activity rhythm. To measure phase shifts, the time between onset of the fused bout and either the dissociated bout onset or the entrained bout onset (data not shown) was measured.

Statistical analysis. Behavioral rhythms in LD22 and DD were statistically evaluated via the $\chi^{2}$ periodogram (Sokolove and Bushell, 1978) using El Temps (A. Diez-Noguera, Barcelona, Spain). Relative OD values for each subregion and phase shifts were statistically evaluated via a $2 \times$ 2 ANOVA with phase (aligned vs misaligned) and light treatment (light pulse vs control) as main effects. c-Fos-immunoreactive cell counts were evaluated using a two-way ANOVA comparing region (vlSCN vs dm$\mathrm{SCN}$ ) and treatment (light pulse vs control). Tukey's post hoc tests were used to evaluate significant effects in ANOVA analyses as appropriate. Phase clustering in control animals was evaluated using the Rayleigh's analysis program (R. Refinetti, University of South Carolina, Walterboro, SC). All results were considered significant at the $p=0.05$ level.

\section{Results}

All experimental animals maintained clear desynchrony of locomotor rhythms in LD22, confirmed by visual inspection and $\chi^{2}$ periodogram analysis. Desynchrony manifested as one activity bout that tracked the dark phase of the LD cycle (entrained bout; $T=22 \mathrm{~h}$ ) and a second, longer bout that dissociated from it (dissociated bout; $\tau>24 \mathrm{~h}$ ). Over time, the two bouts transitioned between aligned phases, in which the dissociated bout occurred during the dark phase of the LD cycle and overlapped the entrained activity bout (Fig. $1 A$, bottom oval), and misaligned phases, in which the dissociated bout occurred during the light phase of the LD cycle, in antiphase to the entrained bout (Fig. $1 A$, top oval). This pattern was consistent with previously reported observations (Campuzano et al., 1998; de la Iglesia et al., $2004 \mathrm{~b}$ ). Animals that failed to desynchronize or that lost the dualperiod pattern while in LD22 were removed from the study before light treatment and are not included in any reported $n$ values. Actograms were inspected daily by an investigator, and animals were scheduled for light treatment $3.8 \mathrm{~h}$ after lights off, at either a maximally aligned phase (both bouts fully coincided) (Fig. 1 A) or a maximally misaligned phase (bouts were $180^{\circ}$ out-of-phase). Pulse schedules were confirmed within $24-48 \mathrm{~h}$ of treatment to ensure accuracy of the predicted phase.

In the first experiment, animals were returned to their home chamber after conclusion of the light pulse and were killed $60 \mathrm{~min}$ after the start of the pulse $(n=23)$. We asked whether light pulses
A

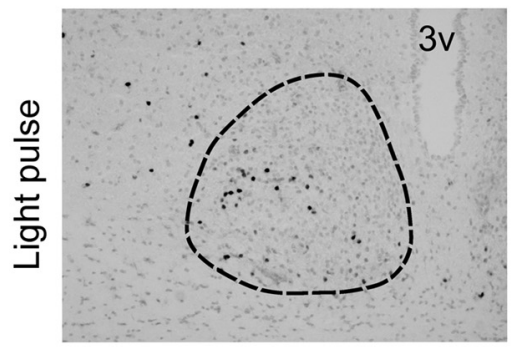

B

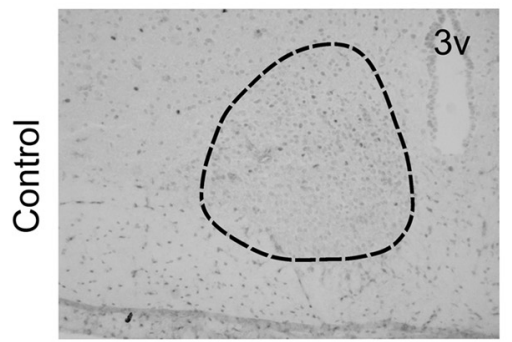

C

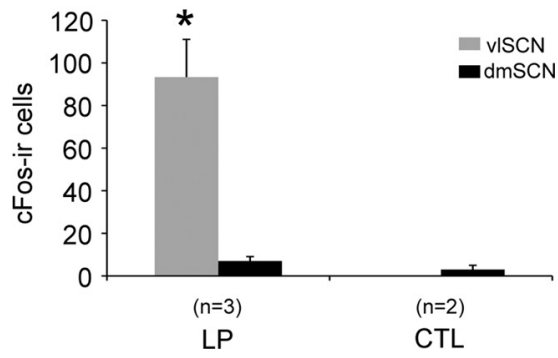

Figure 2. Light pulses induce c-Fos expression within the vISCN regardless of the dmSCN circadian phase. $\boldsymbol{A}, \boldsymbol{B}$, Representative photomicrographs of $\mathrm{c}-\mathrm{Fos}$-immunoreactive cells in the $\mathrm{SCN}$ (outlined) of misaligned phase rats after light pulse $(\boldsymbol{A} ; \mathrm{LP})$ or control dark pulse ( $\boldsymbol{B} ; \mathrm{CTL}) . \boldsymbol{C}$, Counts of c-Fos-immunoreactive cells in the vISCN and dmSCN; ${ }^{*} p=0.02$ (compared with all other groups).

would induce Perl expression in the desynchronized SCN via in situ hybridization autoradiography for Perl mRNA. In the vlSCN, light pulses increased Per1 expression compared with control animals $\left(F_{(1,19)}=28.51, p<0.0001\right)$ (Fig. $\left.1 B, C\right)$, and there was neither a main effect of phase of treatment $\left(F_{(1,19)}=\right.$ 2.36, $p=0.14$ ) nor an interaction between the two factors (light treatment and phase $)\left(F_{(1,19)}=0.57, p=0.46\right)$. In contrast, light had no effect on dmSCN per1 expression $\left(F_{(1,19)}=1.75, p=\right.$ $0.20)$. As expected, Per $1 \mathrm{mRNA}$ expression varied as a function of phase $\left(F_{(1,19)}=18.45, p=0.0004\right)$ (Fig. $\left.1 B, D\right)$, with high Per1 expression during the dmSCN subjective day (misaligned phase) and low during the dmSCN subjective night (aligned phase). There was no interaction between treatment and phase in the $\operatorname{dmSCN}\left(F_{(1,19)}=1.44, p=0.25\right)$. Thus, nighttime light pulses induced Perl expression in the vlSCN, a critical component of the phase-resetting response of the pacemaker, regardless of the phase of the dmSCN. In contrast, Perl expression in the dmSCN solely reflected its spontaneous oscillation and was independent of the light treatment.

We next asked whether the response to light pulses was preserved at the protein level by assessing c-Fos immunohistochemistry $(n=5)$. In animals killed at their maximally misaligned phase, c-Fos-IR was elevated after light pulses in the vISCN relative to control animals (significant interaction between light treatment and subregion, $F_{(1,3)}=19.51, p=0.02$ ) (Fig. 2). In contrast, c-Fos-IR in the dmSCN remained low in both light- 
A

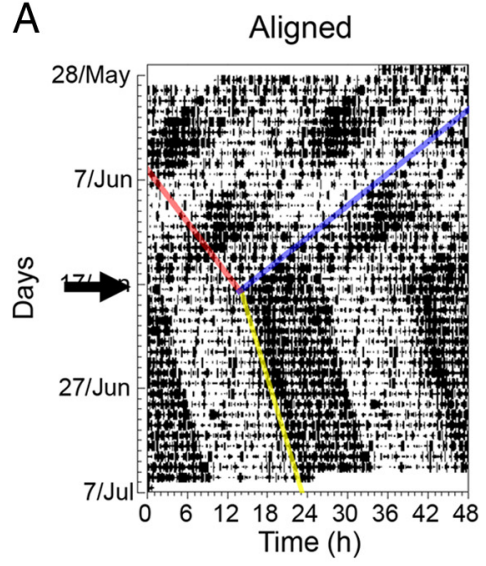

C

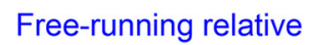
to entrained onset

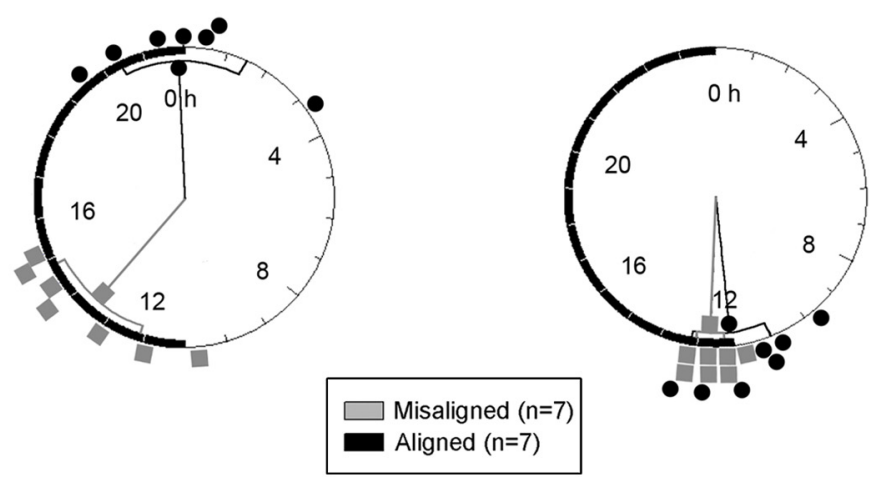

Figure 3. The phase of circadian locomotor activity after release of LD22 desynchronized rats into DD. $A, B$, Representative double-plotted actograms of aligned-phase $(\boldsymbol{A})$ and misaligned-phase $(\boldsymbol{B})$ rats released into DD after a dark pulse (arrows), showing the eye-fitted onsets of the entrained locomotor onset (blue line), the dissociated locomotor bout (red line), and the fused, free-running locomotor bout (yellow line). $C, D$, Rayleigh phase plots showing the fused locomotor bout onsets for alignedphase (gray squares) and misaligned-phase (black circles) animals, extrapolated to the day of control pulse. Onsets are referenced to the entrained bout onset and plotted on a $22 \mathrm{~h}$ timescale $(\boldsymbol{C})$ or referenced to the dissociated bout onset, plotted on a $25 \mathrm{~h}$ timescale, which approximates the average period of the dissociated locomotor bout $(\boldsymbol{D})$.

pulsed and control animals. Thus, two classical markers of the phase-specific response of the SCN to light, Perl mRNA and c-Fos-IR, were upregulated by nighttime light pulses in the retinorecipient vlSCN even when these light pulses were administered during the dmSCN subjective day.

To assess whether the responses to light pulses observed within the SCN would translate into a phase shift of circadian locomotor rhythms, a second set of animals $(n=27)$ was desynchronized, exposed to light pulses or control dark pulses, and released into DD immediately after light treatment. Desynchronized activity bouts rapidly fused after release into DD and regained a stable onset and offset within $7 \mathrm{~d}$ of release into $\mathrm{DD}$ in all animals (Fig. $3 A, B$ ). These free-running activity onsets were extrapolated back to the day of the light treatment. Rayleigh's analyses of dark-pulsed animals detected significant clustering of phases of this free-running bout in aligned- and misalignedphase animals when measured relative to the entrained activity bout onset (i.e., lights off) on a $22 \mathrm{~h}$ timescale (the period of the entrained activity bout) (aligned, $\chi^{2}=13.805, p=0.001$; misaligned, $\chi^{2}=12.268, p=0.002$ ) (Fig. 3C). The same test detected significant clustering of phases relative to the dissociated activity onset (aligned, $\chi^{2}=14.000, p<0.001$; misaligned, $\chi^{2}=13.883$, $p=0.001$ ) (Fig. 3D) in the last day under LD22. However, onsets plotted relative to the entrained bout exhibited a biphasic distri- bution with significantly different mean phases $\left(t_{(12)}=9.224, p<0.001\right)$. This analysis revealed that, although the phase of aligned animals was close to the time of lights off on the $22 \mathrm{~h}$ cycle, the phase of the misaligned animals was close to the time of lights on. In contrast, free-running onsets plotted relative to the dissociated bout onset on a $25 \mathrm{~h}$ timescale (the period of the dissociated activity bout) uniformly clustered around the dissociated bout onset in both aligned and misaligned-phase animals $\left(t_{(12)}=-1.960, p=0.074\right)$. Thus, in the forced desynchronized rat, the phase of the free-running activity rhythm after release of dark-pulsed animals into DD was predicted by the dissociated activity bout, not the $22 \mathrm{~h}$ bout; for this reason, we measured phase shifts relative to the dissociated bout onset. The clustering of phases relative to the entrained activity rhythm was reported previously by Campuzano et al. (1998), who also found an odd phase relationship with the LD cycle. This clustering relative to the $\mathrm{LD}$ cycle suggested that the $22 \mathrm{~h} \mathrm{LD}$ cycle, and by extension the vlSCN, could set the phase of the free-running rhythm; however, in that study, the authors did not analyze the clustering relative to the dissociated rhythm. Our purposely designed release into DD from aligned and misaligned phases clearly shows that the dissociated rhythm is the predominant determinant of the free-running phase.

Light pulses administered during the early part of the dark phase induced a large phase delay $(\sim 2 \mathrm{~h})$ in aligned-phase animals only (Fig. $4 A$ ), with onsets in all other groups within $30 \mathrm{~min}$ of the last dissociated bout onset (Figs. $3 A, B, 4 B$ ). There was a significant main effect of phase of treatment $\left(F_{(1,23)}=19.23, p=0.0002\right)$ and a significant interaction $\left(F_{(2,23)}=4.15, p=0.05\right)$ (Fig. $\left.4 C\right)$, such that aligned-phase animals receiving a light pulse showed a significant phase shift ( $p<0.0096$ vs all other groups, Tukey's contrasts), although no other significant differences between groups were detected. Thus, light pulses delivered during the night of vlSCN induced a phaseresetting response in the vlSCN as reflected by induction of Per 1 mRNA and c-Fos-IR and did so independently of the phase of the dmSCN. However, the same light pulse failed to shift locomotor activity if the dmSCN was in its subjective day.

\section{Discussion}

The phase-specific response of the circadian pacemaker to light constitutes the basis for photic entrainment of circadian rhythms (Pittendrigh and Daan, 1976; Johnson et al., 2003). Although the anatomical and neurochemical circuits by which light stimulates the SCN are well described (Morin and Allen, 2006), the mechanisms underlying the phase specificity of its response are still unclear. The complex anatomy of the SCN is thought to be critical for processing phase-resetting signals (Antle and Silver, 2005). However, vlSCN efferents traverse the dmSCN (Leak et al., 1999; Leak and Moore, 2001), retinorecipient vlSCN cells relay 

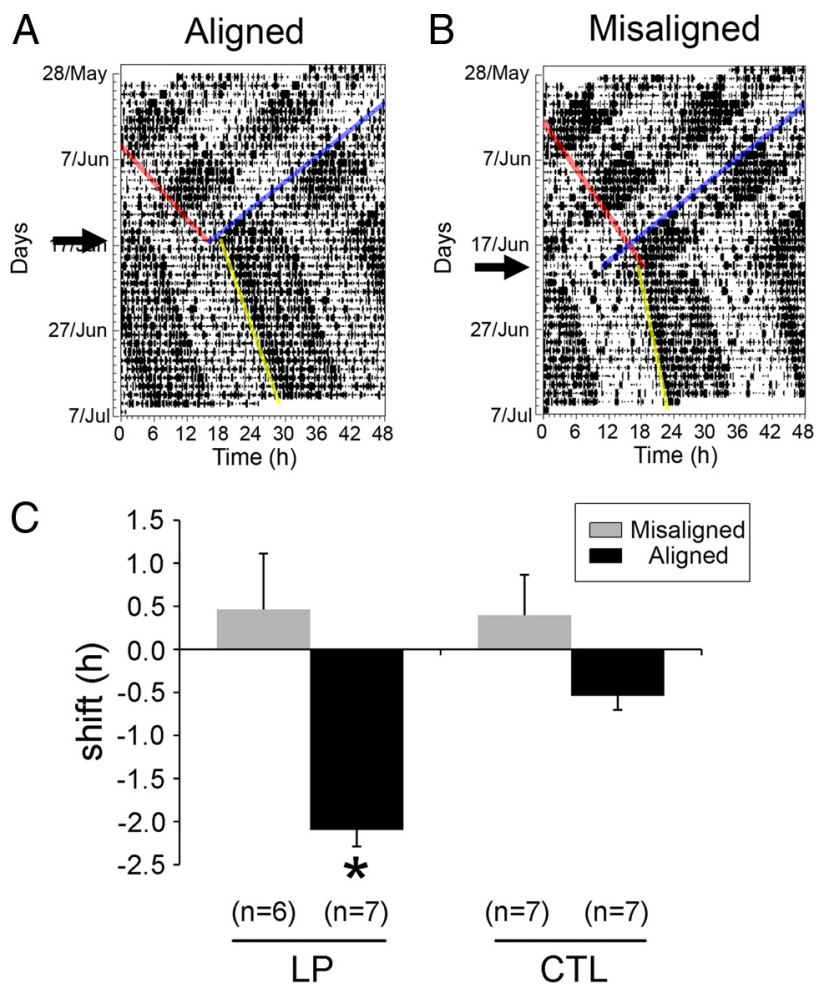

Figure 4. $\boldsymbol{A}, \boldsymbol{B}$, Representative double-plotted actograms of aligned-phase $(\boldsymbol{A})$ and misaligned-phase $(\boldsymbol{B})$ rats released into DD after a light pulse (arrows), showing the eye-fitted onsets of the entrained locomotor onset (blue line), the dissociated locomotor bout (red line), and the fused, free-running locomotor bout (yellow line). C, Light pulses induce phase shifts of circadian locomotor activity only when there is phase alignment between the vISCN and dmSCN cells. Phase shift in hours for rats given light pulses (LP) or control dark pulses (CTL) and then released into DD. ${ }^{*} p=0.05$.

photic information to second-order cells, and the SCN is itself small, rendering the study of the contributions of the SCN subregions to photic decoding difficult when using conventional techniques such as neuroanatomical lesions. Here, we capitalized on the steady-state dissociation of vISCN and dmSCN oscillators in the forced desynchronized rat (de la Iglesia et al., 2004b; Lee et al., 2009) to assess whether the night-specific response to light depends on synchrony between neuronal oscillators within the SCN. Our results indicate that the phase-specific induction of Per1 mRNA and c-Fos protein within the vlSCN does not depend on circadian timing information from the $\mathrm{dmSCN}$ and that this acute induction of gene expression is only associated with phase shifts when the two subregions are phase aligned.

The acute induction of Per1 mRNA (Shigeyoshi et al., 1997; Yan et al., 1999; Hamada et al., 2001; de la Iglesia et al., 2004a; Karatsoreos et al., 2004), c-fos mRNA (Kornhauser et al., 1990; Schwartz et al., 2000), and c-Fos protein (Schwartz et al., 1994; Sumová et al., 1998) after light pulses is localized to the retinorecipient vlSCN or "core," even in species in which the dorsal SCN receives ipsilateral retinal input (Muscat et al., 2003; Morin and Allen, 2006). In all species, light-induced increases in gene expression only occur when pulses are presented at night (Kornhauser et al., 1990; Rusak et al., 1990; Shigeyoshi et al., 1997; Miyake et al., 2000); however, the mechanism underlying this temporal regulation is poorly understood. Several models propose that light entrains an oscillator in the retinorecipient vlSCN, which in turn entrains a second oscillator in the dmSCN (Shigeyoshi et al., 1997; Schwartz et al., 2009). Alternatively, the retinorecipient SCN could be non-oscillatory, and its circadian- gated response could be driven by information from SCN oscillator neurons elsewhere (Antle et al., 2003, 2007). In support of this idea, light-responsive SCN neurons immunopositive for calbindin (hamster) or gastrin-releasing peptide (mouse) do not exhibit spontaneous rhythms in firing rate (Jobst and Allen, 2002) or Per1 expression (Hamada et al., 2001; Kuhlman et al., 2003; Karatsoreos et al., 2004). In the present study, light pulses administered during the dark phase of the $22 \mathrm{~h}$ LD cycle increased Per 1 mRNA expression and c-Fos-IR in the vlSCN. In contrast, in the dmSCN, Perl expression tracked the dissociated locomotor bout, and light pulses affected neither Per1 expression nor c-FosIR. The vISCN thus mounted a photic response regardless of whether the dmSCN was in its subjective night (low Per1 expression) or its subjective day (high Per1 expression). In forced desynchronized rats, clock gene expression in the vlSCN tracks the LD cycle, and the light-phase peak in Per1 expression persists in vivo after release into DD (de la Iglesia et al., 2004b), as well as in vitro as reported by Per1-driven luciferase activity (M.D.S., unpublished observations). Thus, the vlSCN indeed can, at least transiently, oscillate in the absence of light input, consistent with the fact that virtually all SCN neurons, regardless of their neurotransmitter phenotype, can oscillate autonomously (Aton and Herzog, 2005; Webb et al., 2009). Increased Per1 expression during the vlSCN subjective day precludes evaluation of the response to light pulses during this phase, a condition that should be met if indeed the vlSCN gates its own light response. The present data do not rule out the possibility that the misaligned-phase response within the vlSCN may itself differ in some way from the aligned-phase response. Detailed characterization of the photic response using established neuroanatomical markers could clarify its extent and perhaps its functional basis in aligned compared with misaligned phases. Nevertheless, the vlSCN clearly regulates its nighttime response to light independently of the phase of the dmSCN, consistent with our model of SCN subregional decoding of light information in the rat (Schwartz et al., 2009).

Under LD22, rhythms in REM sleep, core body temperature, and pineal melatonin release express a single rhythm with a period longer than $24 \mathrm{~h}$ (Cambras et al., 2007; Schwartz et al., 2009), and the dissociated REM sleep rhythm tracks Per1 expression in the dmSCN (Lee et al., 2009), suggesting that the dmSCN could determine the phase for a significant subset of SCN outputs. In the present study, after release into DD, the fused locomotor activity bout free-ran from the last dissociated bout onset. In other words, regardless of whether animals were released from aligned or misaligned phases, the phase of the free-running rhythm after release into DD clustered around the last dissociated bout onset. At aligned phases, clock gene expression is similar in the dmSCN and vlSCN (de la Iglesia et al., 2004b); one would thus expect locomotor activity to free-run in DD based on the phase of this consolidated SCN. Conversely, at misaligned phases, clock gene expression is $180^{\circ}$ out-of-phase in the dmSCN and vlSCN (de la Iglesia et al., 2004b), raising the possibility that, after release, the net output of the SCN could be based on the phase of either subregion or on some compromise between them. We conclude that the dmSCN sets the phase for the output of the resynchronized SCN because the fused locomotor rhythm consistently reflected the phase of the dissociated bout after release into DD. Similarly, the initial phase of pineal melatonin rhythms in DD matches the phase of the dissociated activity bout under LD22, suggesting that both rhythms are coded by a common underlying subregional oscillator (Schwartz et al., 2009). 
The anatomy of the SCN and existing functional data strongly suggest that the pacemaker processes information hierarchically. Direct and indirect retinal input is concentrated in the vlSCN, whereas hypothalamic and limbic input is concentrated in the dmSCN (Moga and Moore, 1997); connections between the two subregions are predominantly ventral to dorsal (Leak et al., 1999; Kriegsfeld et al., 2004), and the efferent projections of the SCN are partially segregated by subregion (Abrahamson and Moore, 2001; Leak and Moore, 2001; Kriegsfeld et al., 2004). Administration of two light pulses $1 \mathrm{~h}$ apart (for review, see Daan and Aschoff, 2001) reveals that the second light pulse induces gene expression in the vlSCN but fails to shift behavioral rhythms (Best et al., 1999), suggesting that shifts result from integration between readily light-responsive vlSCN cells and nonresponsive $\mathrm{dmSCN}$ cells. Consistent with this, Per-luciferase bioluminescence rhythms (Nakamura et al., 2005; Davidson et al., 2009) and firing rhythms reset faster in the ventral than in the dorsal SCN (Albus et al., 2005), suggesting that phase-resetting cues are processed first by the vlSCN and then relayed to the dmSCN (Shigeyoshi et al., 1997; Kuhlman et al., 2003). Our results support this in three ways. First, Perl expression and c-Fos-IR in the vlSCN always responded to light pulses delivered at night regardless of the phase of the dmSCN, consistent with an initial vlSCNlocalized processing of light cues that does not require dmSCN input. Second, in control-pulsed rats, the fused locomotor bout free-ran in DD from the last onset of the dissociated bout whether that bout was aligned with the LD cycle or not, showing that the $\mathrm{dmSCN}$ constitutes a key regulator of SCN outputs. Finally, light pulses only phase-shifted the fused locomotor rhythm when the $\mathrm{dmSCN}$ was in its subjective night, suggesting that the dmSCNcontrolled activity onset is sensitive to signals from the lightresponsive vlSCN in a phase-dependent manner. Alternatively, the response to light by dmSCN could be independent from light input to the vlSCN.

The present study shows that the nighttime acute photic induction of Perl in the vlSCN can be functionally dissociated from the phase-resetting of pacemaker outputs, measured by phase shifting of locomotor rhythms. Together, these results are consistent with a model in which light entrains a retinorecipient vlSCN oscillator, which in turn entrains a second oscillator located in the dmSCN (Schwartz et al., 2009). This model is based on the anatomy of the SCN (Moore et al., 2002; Morin et al., 2006), on previous models of SCN organization (Shigeyoshi et al., 1997), and on the characterization of SCN outputs under LD22 (de la Iglesia et al., 2004b; Cambras et al., 2007; Lee et al., 2009; Schwartz et al., 2009). Our model proposes that each of these two oscillators has its own phase-response curve (Schwartz et al., 2009) and predicts that the phase misalignment between neuronal oscillators induced by stable forced desynchrony (de la Iglesia et al., 2004b; Lee et al., 2009) positions the vlSCN in a light-sensitive phase (circadian night) and the $\mathrm{dmSCN}$ in a lightinsensitive phase (circadian day). Our model predicts as well that the transient internal desynchronization that results from jetlag (Albus et al., 2005; Nakamura et al., 2005; Davidson et al., 2009) would lead to a similar ability of light to induce acute gene expression in the master circadian pacemaker but inability to induce behavioral phase shifts.

\section{References}

Abrahamson EE, Moore RY (2001) Suprachiasmatic nucleus in the mouse: retinal innervation, intrinsic organization and efferent projections. Brain Res 916:172-191.

Albus H, Vansteensel MJ, Michel S, Block GD, Meijer JH (2005) A GABAergic mechanism is necessary for coupling dissociable ventral and dorsal regional oscillators within the circadian clock. Curr Biol 15:886-893.

Antle MC, Silver R (2005) Orchestrating time: arrangements of the brain circadian clock. Trends Neurosci 28:145-151.

Antle MC, Foley DK, Foley NC, Silver R (2003) Gates and oscillators: a network model of the brain clock. J Biol Rhythms 18:339-350.

Antle MC, Foley NC, Foley DK, Silver R (2007) Gates and oscillators II: zeitgebers and the network model of the brain clock. J Biol Rhythms 22:14-25.

Aronin N, Sagar SM, Sharp FR, Schwartz WJ (1990) Light regulates expression of a Fos-related protein in rat suprachiasmatic nuclei. Proc Natl Acad Sci U S A 87:5959-5962.

Aton SJ, Herzog ED (2005) Come together, right ... now: synchronization of rhythms in a mammalian circadian clock. Neuron 48:531-534.

Best JD, Maywood ES, Smith KL, Hastings MH (1999) Rapid resetting of the mammalian circadian clock. J Neurosci 19:828-835.

Cambras T, Weller JR, Anglès-Pujoràs M, Lee ML, Christopher A, DíezNoguera A, Krueger JM, de la Iglesia HO (2007) Circadian desynchronization of core body temperature and sleep stages in the rat. Proc Natl Acad Sci U S A 104:7634-7639.

Campuzano A, Vilaplana J, Cambras T, Díez-Noguera A (1998) Dissociation of the rat motor activity rhythm under T cycles shorter than 24 hours. Physiol Behav 63:171-176.

Daan S, Aschoff J (2001) The entrainment of circadian systems. In: Handbook of behavioral neurobiology: circadian clocks (Takahashi JS, Turek F, eds), pp 7-42. New York: Kluwer Academic/Plenum Publishers.

Davidson AJ, Castanon-Cervantes O, Leise TL, Molyneux PC, Harrington ME (2009) Visualizing jet lag in the mouse suprachiasmatic nucleus and peripheral circadian timing system. Eur J Neurosci 29:171-180.

de la Iglesia HO (2007) In situ hybridization of suprachiasmatic nucleus slices. In: Methods of Molecular Biology (Rosato E, ed), pp 513-531. Totowa, NJ: Humana.

de la Iglesia HO, Meyer J, Schwartz WJ (2004a) Using Per gene expression to search for photoperiodic oscillators in the hamster suprachiasmatic nucleus. Brain Res Mol Brain Res 127:121-127.

de la Iglesia HO, Cambras T, Schwartz WJ, Díez-Noguera A (2004b) Forced desynchronization of dual circadian oscillators within the rat suprachiasmatic nucleus. Curr Biol 14:796-800.

Hamada T, LeSauter J, Venuti JM, Silver R (2001) Expression of Period genes: rhythmic and nonrhythmic compartments of the suprachiasmatic nucleus pacemaker. J Neurosci 21:7742-7750.

Jobst EE, Allen CN (2002) Calbindin neurons in the hamster suprachiasmatic nucleus do not exhibit a circadian variation in spontaneous firing rate. Eur J Neurosci 16:2469-2474.

Johnson CH, Elliott JA, Foster R (2003) Entrainment of circadian programs. Chronobiol Int 20:741-774.

Karatsoreos IN, Yan L, LeSauter J, Silver R (2004) Phenotype matters: identification of light-responsive cells in the mouse suprachiasmatic nucleus. J Neurosci 24:68-75.

Klein DC, Moore RY, Reppert SM (1991) Suprachiasmatic nucleus. The mind's clock. New York: Oxford UP.

Kornhauser JM, Nelson DE, Mayo KE, Takahashi JS (1990) Photic and circadian regulation of $\mathrm{c}$-fos gene expression in the hamster suprachiasmatic nucleus. Neuron 5:127-134.

Kriegsfeld LJ, Leak RK, Yackulic CB, LeSauter J, Silver R (2004) Organization of suprachiasmatic nucleus projections in Syrian hamsters $(\mathrm{Me}-$ socricetus auratus): an anterograde and retrograde analysis. J Comp Neurol 468:361-379.

Kuhlman SJ, Silver R, Le Sauter J, Bult-Ito A, McMahon DG (2003) Phase resetting light pulses induce Perl and persistent spike activity in a subpopulation of biological clock neurons. J Neurosci 23:1441-1450.

Leak RK, Moore RY (2001) Topographic organization of suprachiasmatic nucleus projection neurons. J Comp Neurol 433:312-334.

Leak RK, Card JP, Moore RY (1999) Suprachiasmatic pacemaker organization analyzed by viral transynaptic transport. Brain Res 819:23-32.

Lee ML, Swanson BE, de la Iglesia HO (2009) Circadian timing of REM sleep is coupled to an oscillator within the dorsomedial suprachiasmatic nucleus. Curr Biol 19:848-852.

Miyake S, Sumi Y, Yan L, Takekida S, Fukuyama T, Ishida Y, Yamaguchi S, Yagita K, Okamura H (2000) Phase-dependent responses of Per1 and Per2 genes to a light-stimulus in the suprachiasmatic nucleus of the rat. Neurosci Lett 294:41-44. 
Moga MM, Moore RY (1997) Organization of neural inputs to the suprachiasmatic nucleus in the rat. J Comp Neurol 389:508-534.

Moore RY, Lenn NJ (1972) A retinohypothalamic projection in the rat. J Comp Neurol 146:1-14.

Moore RY, Speh JC, Leak RK (2002) Suprachiasmatic nucleus organization. Cell Tissue Res 309:89-98.

Morin LP (2007) SCN organization reconsidered. J Biol Rhythms 22:3-13.

Morin LP, Allen CN (2006) The circadian visual system, 2005. Brain Res Rev 51:1-60.

Morin LP, Shivers KY, Blanchard JH, Muscat L (2006) Complex organization of mouse and rat suprachiasmatic nucleus. Neuroscience 137: $1285-1297$.

Muscat L, Huberman AD, Jordan CL, Morin LP (2003) Crossed and uncrossed retinal projections to the hamster circadian system. J Comp Neurol 466:513-524.

Nakamura W, Yamazaki S, Takasu NN, Mishima K, Block GD (2005) Differential response of Period 1 expression within the suprachiasmatic nucleus. J Neurosci 25:5481-5487.

Pittendrigh CS, Daan S (1976) Functional-analysis of circadian pacemakers in nocturnal rodents. 4. Entrainment: pacemaker as clock. J Comp Physiol 106:291-331.

Reppert SM, Weaver DR (2002) Coordination of circadian timing in mammals. Nature 418:935-941.

Rusak B, Robertson HA, Wisden W, Hunt SP (1990) Light pulses that shift rhythms induce gene expression in the suprachiasmatic nucleus. Science 248:1237-1240.

Schwartz MD, Wotus C, Liu T, Friesen WO, Borjigin J, Oda GA, de la Iglesia HO (2009) Dissociation of circadian and light inhibition of melatonin release through forced desynchronization in the rat. Proc Natl Acad Sci U S A 106:17540-17545.

Schwartz WJ (2009) Circadian rhythms: a tale of two nuclei. Curr Biol 19:R460-R462.
Schwartz WJ, Takeuchi J, Shannon W, Davis EM, Aronin N (1994) Temporal regulation of light-induced Fos and Fos-like protein expression in the ventrolateral subdivision of the rat suprachiasmatic nucleus. Neuroscience 58:573-583.

Schwartz WJ, Carpino A Jr, de la Iglesia HO, Baler R, Klein DC, Nakabeppu Y, Aronin N (2000) Differential regulation of fos family genes in the ventrolateral and dorsomedial subdivisions of the rat suprachiasmatic nucleus. Neuroscience 98:535-547.

Shigeyoshi Y, Taguchi K, Yamamoto S, Takekida S, Yan L, Tei H, Moriya T, Shibata S, Loros JJ, Dunlap JC, Okamura H (1997) Light-induced resetting of a mammalian circadian clock is associated with rapid induction of the mPer1 transcript. Cell 91:1043-1053.

Sokolove PG, Bushell WN (1978) The chi square periodogram: its utility for analysis of circadian rhythms. J Theor Biol 72:131-160.

Summer TL, Ferraro JS, McCormack CE (1984) Phase-response and Aschoff illuminance curves for locomotor activity rhythm of the rat. Am J Physiol 246:R299-R304.

Sumová A, Trávnícková Z, Mikkelsen JD, Illnerová H (1998) Spontaneous rhythm in c-Fos immunoreactivity in the dorsomedial part of the rat suprachiasmatic nucleus. Brain Res 801:254-258.

Sundquist SJ, Nisenbaum LK (2005) Fast Fos: rapid protocols for singleand double-labeling c-Fos immunohistochemistry in fresh frozen brain sections. J Neurosci Methods 141:9-20.

Webb AB, Angelo N, Huettner JE, Herzog ED (2009) Intrinsic, nondeterministic circadian rhythm generation in identified mammalian neurons. Proc Natl Acad Sci U S A 106:16493-16498.

Welsh DK, Logothetis DE, Meister M, Reppert SM (1995) Individual neurons dissociated from rat suprachiasmatic nucleus express independently phased circadian firing rhythms. Neuron 14:697-706.

Yan L, Takekida S, Shigeyoshi Y, Okamura H (1999) Per1 and Per2 gene expression in the rat suprachiasmatic nucleus: circadian profile and the compartment-specific response to light. Neuroscience 94:141-150. 